УДК 517.958:539.3

\title{
Exact Analytical Solution of One Problem on Planar Deformation of Nonlinear-Elastic Media
}

\author{
Georgiy M. Sevastyanov* \\ Institute of Machine Engineering and Metallurgy FEB RAS \\ Metallurgov, 1, Komsomolsk-on-Amur, 681005
}

Russia

Received 10.05.2015, received in revised form 15.06.2015, accepted 10.07.2015

An analytical solution of a problem on planar deformation of isotropic incompressible nonlinear-elastic (rubber-like) media with a cylindrical cavity is constructed in quasi-static approximation. A contour of the cavity is a smooth symmetrical curve. The special kind of follower load provides purely radial movement of material. Mass forces are neglected. A physical model of medium is given by elastic potential, which is analogous of Mooney-Rivlin strain energy potential (with a difference in the used finite strain tensor). The obtained solution is exact since in equations connecting Cauchy stress tensor and Almansi finite strain tensor all nonlinear terms are kept (for accepted medium model the maximum is the fourth power of components of displacement gradient tensor).

Keywords: planar deformation, nonlinear elasticity, incompressibility, Mooney-Rivlin solid, finite strain, Almansi strain tensor, exact analytical solution.

DOI: $10.17516 / 1997-1397-2015-8-3-352-355$

Let incompressible nonlinear-elastic medium occupies unlimited space and has a cylindrical cutout of infinite length (Fig. 1), on the surface of which the special kind of an uneven follower load (Fig. 2) is given. We assume the contour $R_{0}$ of the cutout border in undeformed state a smooth curve, symmetric about two perpendicular lines. Consider the problem of elastostatic equilibrium of such media on the assumption of plane strain.

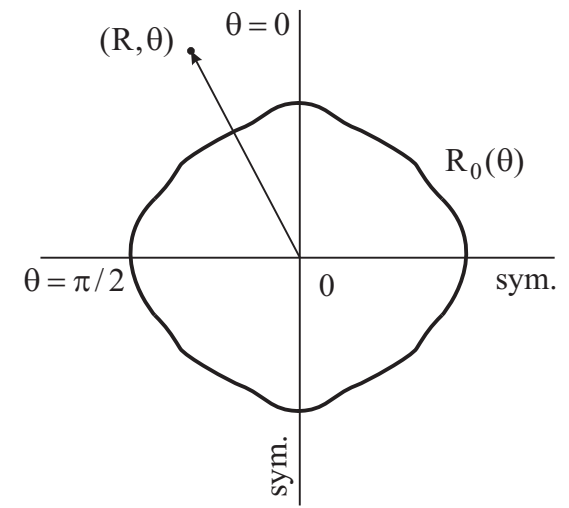

Fig. 1. Computational domain $R(\theta) \geqslant R_{0}(\theta)$

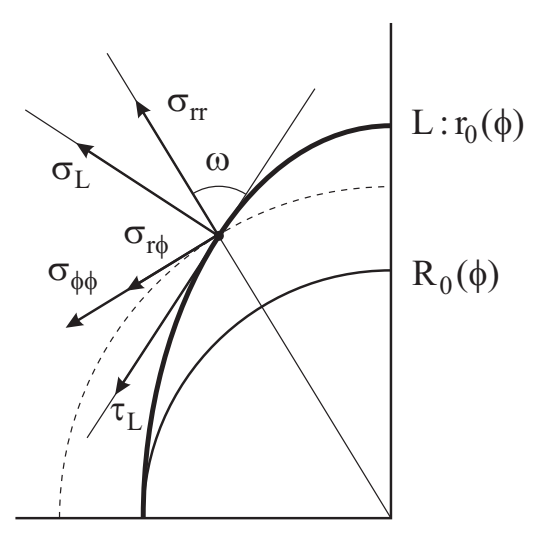

Fig. 2. On the determination of boundary load

*akela.86@mail.ru

(C) Siberian Federal University. All rights reserved 
We introduce a cylindrical coordinate system $(R, \theta, Z)$ centered at the point of intersection of axes of symmetry of the cutout (an axis $Z$ is perpendicular to the plane of the Fig. 1), counting a polar angle $\theta$ will lead from one of the lines of symmetry. Assume that points of continuum are displaced only radially during a loading. We define a motion of continuous medium in the Euler form as $R=r-u_{r}(r, \phi), \theta=\phi, Z=z$, where $(R, \theta, Z)$ are coordinates of points of medium in the initial state, $(r, \phi, z)$ - same in the deformed state.

Almansi finite srain tensor is $\mathbf{A}=\left[(\nabla u)+(\nabla u)^{T}-(\nabla u)^{T}(\nabla u)\right] / 2$, where the displacement gradient tensor in cylindrical coordinates in view of $u \equiv u_{r}(r, \phi)$ has the form

$$
\nabla u \equiv\left(\begin{array}{ccc}
\frac{\partial u_{r}}{\partial r} & \frac{1}{r} \cdot \frac{\partial u_{r}}{\partial \phi} & 0 \\
0 & \frac{u_{r}}{r} & 0 \\
0 & 0 & 0
\end{array}\right)
$$

Its nonzero components are expressed through displacement as follows:

$$
\begin{gathered}
\alpha_{r r}=\frac{1}{2} \cdot \frac{\partial u_{r}}{\partial r} \cdot\left(2-\frac{\partial u_{r}}{\partial r}\right), \quad \alpha_{\phi \phi}=\frac{1}{2} \cdot \frac{u_{r}}{r} \cdot\left(2-\frac{u_{r}}{r}\right)-\frac{1}{2} \cdot\left(\frac{1}{r} \cdot \frac{\partial u_{r}}{\partial \phi}\right)^{2}, \\
\alpha_{r \phi}=\alpha_{\phi r}=\frac{1}{2} \cdot\left(\frac{1}{r} \cdot \frac{\partial u_{r}}{\partial \phi}\right) \cdot\left(1-\frac{\partial u_{r}}{\partial r}\right) .
\end{gathered}
$$

Incompressibility condition is $\left(1-2 \cdot A_{1}\right) \cdot\left(1-2 \cdot A_{2}\right)=1$, where $A_{1}, A_{2}$ are the principal values of the Almansi strain tensor $\left(A_{3}=0\right)$. Integration of the incompressibility equation relative to displacement (assuming $u_{r} / r<1, \partial u_{r} / \partial r<1$ ) gives a view of the solution:

$$
u_{r}(r, \phi)=r-\sqrt{r^{2}+\psi(\phi)}
$$

thus the problem of determining the kinematics of media is reduced to identification of $\psi(\phi)$.

We define physical relations by elastic potential of isotropic incompressible medium (which is analogous to the Mooney-Rivlin potential [1]) in the form $W\left(I_{1}, I_{2}\right)=-b \cdot\left(I_{1}-a \cdot I_{2}\right)$, where $a, b$ is elastic constants, $b>0$ is in pressure units, $a \in(0,2)$ is dimensionless quantity; $I_{1}, I_{2}$ are linear and quadratic invariants of the Almansi tensor, $I_{1}=\operatorname{tr}(\mathbf{A}), 2 \cdot I_{2}=(\operatorname{tr}(\mathbf{A}))^{2}-\operatorname{tr}\left(\mathbf{A}^{2}\right)$.

The components of the Cauchy stress tensor $\boldsymbol{\sigma}$ are determined by dependencies [2] $\sigma_{i j}=-P \cdot \delta_{i j}+\frac{\partial W}{\partial \alpha_{i k}} \cdot\left(\delta_{k j}-2 \cdot \alpha_{k j}\right)$, sum by index $k, P$ is unknown function of additional hydrostatic pressure, $\delta_{i j}$ is Kronecker delta.

In expanded form, in view of $I_{1}=\alpha_{r r}+\alpha_{\phi \phi}, I_{2}=\alpha_{r r} \cdot \alpha_{\phi \phi}-\alpha_{r \phi} \cdot \alpha_{\phi r}, \partial I_{1} / \partial \alpha_{i j}=\delta_{i j}$, $\partial I_{2} / \partial \alpha_{i j}=I_{1} \cdot \delta_{i j}-\alpha_{i j}$ and hence $\partial W / \partial \alpha_{i j}=-b \cdot\left(\delta_{i j}-a \cdot\left(I_{1} \cdot \delta_{i j}-\alpha_{i j}\right)\right)$, we have:

$$
\begin{gathered}
\sigma_{r r}=-P-b \cdot\left(1-a \cdot \alpha_{\phi \phi}\right) \cdot\left(1-2 \cdot \alpha_{r r}\right)+2 \cdot a \cdot b \cdot \alpha_{r \phi}^{2}, \quad \sigma_{r \phi}=b \cdot(2-a) \cdot \alpha_{r \phi}, \\
\sigma_{\phi \phi}=-P-b \cdot\left(1-a \cdot \alpha_{r r}\right) \cdot\left(1-2 \cdot \alpha_{\phi \phi}\right)+2 \cdot a \cdot b \cdot \alpha_{r \phi}^{2} .
\end{gathered}
$$

The equilibrium equations (without mass forces):

$$
\begin{gathered}
\frac{\partial \sigma_{r r}}{\partial r}+\frac{\sigma_{r r}-\sigma_{\phi \phi}}{r}+\frac{1}{r} \cdot \frac{\partial \sigma_{r \phi}}{\partial \phi}=0, \\
\frac{\partial \sigma_{r \phi}}{\partial r}+\frac{1}{r} \cdot \frac{\partial \sigma_{\phi \phi}}{\partial \phi}+\frac{2}{r} \cdot \sigma_{r \phi}=0 .
\end{gathered}
$$

We write the auxiliary relations resulting from (2):

$$
\frac{\partial u_{r}}{\partial r}=-\frac{u_{r}}{r-u_{r}}, \quad \frac{\partial u_{r}}{\partial \phi}=-\frac{1}{2} \cdot \frac{\psi^{\prime}}{r-u_{r}},
$$


then from (1)

$$
\alpha_{r \phi}=-\frac{1}{4} \cdot \frac{\psi^{\prime}}{r^{2}+\psi}, \quad \frac{\partial \alpha_{r \phi}}{\partial r}=\frac{r}{2} \cdot \frac{\psi^{\prime}}{\left(r^{2}+\psi\right)^{2}},
$$

the prime denotes a derivative with respect to $\phi$.

Integrating (5) in view of (6) in relation to $\sigma_{\phi \phi}$, we have:

$$
\sigma_{\phi \phi}=\frac{b \cdot(2-a)}{2} \cdot\left(C(r)+\ln \left(r^{2}+\psi\right)-\frac{\psi}{r^{2}+\psi}\right),
$$

where $C(r)$ is some arbitrary function.

Now the function of additional hydrostatic pressure (and, consequently, the stress component $\left.\sigma_{r r}\right)$ can be expressed from $(3)$ by $C(r)$ and $\psi(\phi)$, and the difference $\left(\sigma_{r r}-\sigma_{\phi \phi}\right)$ and $\partial \sigma_{r \phi} / \partial \phi-$ by $\psi(\phi)$. After the necessary transformations (4) becomes

$$
\frac{1}{2} \cdot \frac{d C}{d r}-\frac{2 \cdot r^{2} \cdot \psi^{\prime \prime}+\psi^{\prime 2}+4 \cdot \psi^{2}-8 \cdot r^{4}}{8 \cdot r^{3} \cdot\left(r^{2}+\psi\right)}=0 .
$$

Differentiating (7) with respect to $\phi$, we have:

$$
2 \cdot r^{4} \cdot\left(\psi^{\prime \prime \prime}+4 \cdot \psi^{\prime}\right)+2 \cdot r^{2} \cdot \psi \cdot\left(\psi^{\prime \prime \prime}+4 \cdot \psi^{\prime}\right)+\psi^{\prime} \cdot\left(2 \cdot \psi \cdot \psi^{\prime \prime}-\psi^{2}+4 \cdot \psi^{2}\right)=0 .
$$

The last equation is divided in the case of $\psi^{\prime} \neq 0$ into two autonomous differential equations with respect to $\psi(\phi)$ :

$$
\left\{\begin{array}{l}
\psi^{\prime \prime \prime}+4 \cdot \psi^{\prime}=0, \\
2 \cdot \psi \cdot \psi^{\prime \prime}-\psi^{2}+4 \cdot \psi^{2}=0 .
\end{array}\right.
$$

Note that $d\left(2 \cdot \psi \cdot \psi^{\prime \prime}-\psi^{\prime 2}+4 \cdot \psi^{2}\right) / d \phi=2 \cdot \psi \cdot\left(\psi^{\prime \prime \prime}+4 \cdot \psi^{\prime}\right)$, hence, any non-trivial solution of the second equation of the system identically satisfies the first equation.

Thus, equation (7) has an exact solution of the form:

$$
\psi(\phi)=k \cdot \cos ^{2}\left(\phi+\phi_{0}\right), \quad C^{\prime}(r)=\left(k-2 \cdot r^{2}\right) / r^{3},
$$

where $k$ and $\phi_{0}$ are constants of integration.

Symmetry conditions $\left.\sigma_{r \phi}\right|_{\phi=0}=\left.\sigma_{r \phi}\right|_{\phi=\pi / 2}=0$ are satisfied by $\phi_{0}=0$ (or $\phi_{0}=\pi / 2$, solutions are identical up to rotation of the coordinate axes through $\pi / 2$ ). In the absence of stress at infinity, we have $C(r)=-\ln \left(r^{2}\right)-k /\left(2 \cdot r^{2}\right)$. The constant of integration $k$ can be considered as time-similar parameter of loading.

Obviously, for compliance with adopted kinematics $\left(u \equiv u_{r}(r, \phi)\right)$ the load on the surface of the cylindrical cutout can not contain functional arbitrariness.

Normal $\sigma_{L}$ and tangent $\tau_{L}$ components of boundary load have the form $\sigma_{L}=\mathbf{n} \cdot \boldsymbol{\sigma} \cdot \mathbf{n}^{T}$, $\tau_{L}=\mathbf{s} \cdot \boldsymbol{\sigma} \cdot \mathbf{n}^{T}$, where the value of the stress tensor $\boldsymbol{\sigma}$ should be taken at the boundary $L: r_{0}(\phi)$ in the actual configuration in view of $r_{0}(\phi)=\sqrt{R_{0}^{2}(\phi)-\psi(\phi)} ; \mathbf{n}=(\sin \omega, \cos \omega, 0)$, $\mathbf{s}=(-\cos \omega, \sin \omega, 0)$ are normal and tangential to $L$ unit vectors, respectively. The function $\omega(\phi)$ is the angle between the extension of the radius-vector and the tangent to $L$, it is defined by the following relationship [3, Ch. VII, §2.232]: $\omega(\phi)=\arctan \left|\frac{r_{0}(\phi)}{r_{0}^{\prime}(\phi)}\right|$.

Consider the case when the contour of the cavity in the undeformed state has the form of an ellipse with semi-axes equal to 1 and $1 / 2: R_{0}(\phi)=1 / \sqrt{\sin ^{2} \phi+4 \cdot \cos ^{2} \phi}$. Let the elastic modulus to be $a=1$. The Fig. 3 shows graphs $\sigma_{L} / b$ and $\tau_{L} / b$ for the loading parameter 


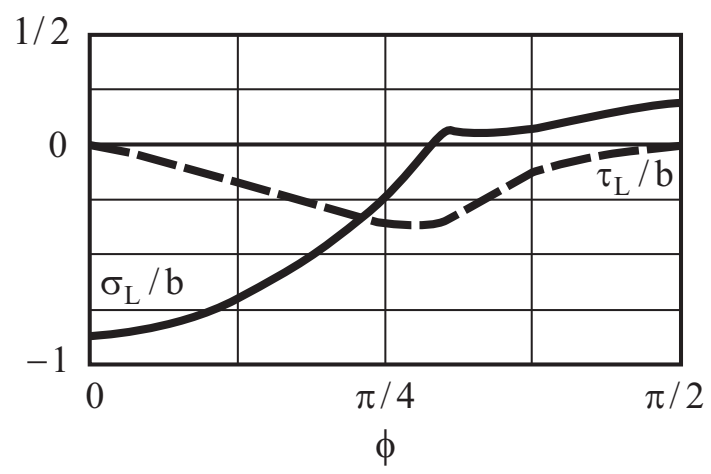

Fig. 3. Components of boundary load

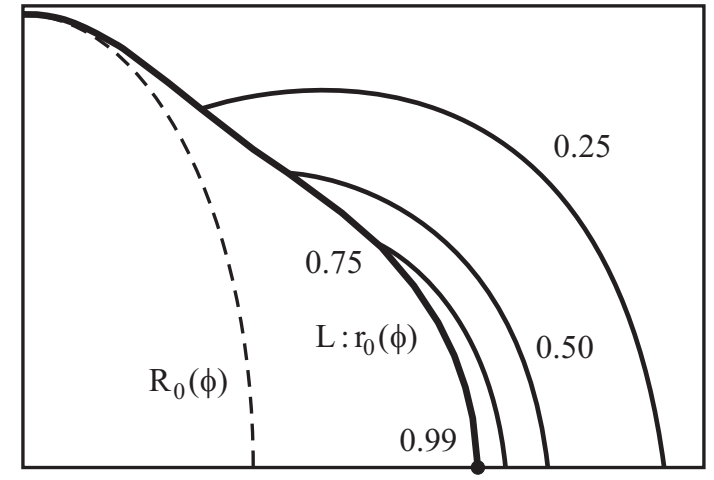

Fig. 4. Stress intensity (von Mises)

value $k=-3 / 4$ (it corresponds to a deformed state with $r_{0}(0)=1$ ). The Fig. 4 shows isolines of tangential stress intensity (according to von Mises), which is also divided by the elastic modulus $b$.

Thus, the maximum stress intensity falls on the point at the boundary of computational domain with polar angle $\phi=0$. From this point the propagation of elasto-plastic boundary begins, when a load reaches some critical value.

Obtained solution can be useful for testing algorithms and programs for numerical integration of equations of the nonlinear theory of elasticity.

\title{
References
}

[1] M.Mooney, A theory of large elastic deformation, J. of App. Phys., 9(1940), no. 11, 582-592.

[2] A.I.Lurie, Nonlinear theory of elasticity, Amsterdam, North-Holland, 1990.

[3] G.M.Fikhtengolts, Calculus, V.1, M., Fizmatlit, 1962 (in Russian).

\section{Точное аналитическое решение одной задачи о плоской деформации нелинейно-упругой среды}

Георгий М. Севастьянов

\begin{abstract}
Построено аналитическое решение задачи о плоской деформачии изотропной нелинейно-упругой несжимаемой (резиноподобной) средъ с иилиндрической полостъю в квазистатическом приближении. Контур полости представляет собой гладкую симметричную кривую. Определенный частный вид следящего нагружения обеспечивает чисто радиальное перемещение материала. Массовые силы не учитываются. Физическая модель среды задана упругим потенииалом, аналогичным потенциалу Муни-Ривлина (с различием в используемом тензоре конечных деформаций). Полученное решение является точным: в уравнениях связи тензоров напряжений Коши и конечных деформачий Альманси сохранены все нелинейные члены (максимальная степень при выбранной модели среды - четвертая по компонентам градиента вектора перемещений).
\end{abstract}

Ключевые слова: плоская деформачия, нелинейная упругость, несжимаемость, среда МуниРивлина, конечные деформачии, тензор Альманси, точное аналитическое решение. 